\title{
Conocimientos, actitudes y prácticas de los matarifes acerca de la hidatidosis/equinococosis, en dos zonas urbanas del Departamento de Ica, Perú
}

\author{
Rufino Cabrera ${ }^{1}$, Eduardo Talavera ${ }^{2}$, María del Pilar Trillo-Altamirano ${ }^{2}$
}

Resumen

\begin{abstract}
Objetivos: Determinar los conocimientos, actitudes y prácticas (CAP) acerca de Echinococcus granulosus e hidatidosis/equinococosis en matarifes municipales de zonas urbanas del Departamento de Ica, Perú. Materiales y Métodos: Estudio observacional, descriptivo. Cincuenta y cinco trabajadores de la ciudad de Ica y Chincha fueron entrevistados anónimamente acerca de E. granulosus e hidatidosis/ equinococosis, en diciembre de 2000. Resultados: El metacestodo de E. granulosus fue conocido como 'bolsa de agua'por 25 (45,5\%) de los entrevistados y como quiste hidatídico por 10 (18,2\%). Los vacunos y ovinos fueron identificados como los huéspedes intermediarios más frecuentes; sin embargo, no conocían los huéspedes definitivos ni los mecanismos de transmisión. La actitud de 36 (65,5\%) matarifes, si estuvieran infectados, era tratarse y, si observaran helmintos en las heces de sus perros, 17 (30,9\%) los sacrificarían. Veintiuno (38,2\%) de los trabajadores admitieron que los quistes hidatídicos van al piso del camal, 30 (54,5\%) criaban perros, 19 $(63,3 \%)$ no los habían desparasitado en los últimos seis meses. Treinta (54,5\%) de los matarifes realizaban beneficios clandestinos y algunos alimentaban a sus perros con vísceras parasitadas crudas. Conclusiones: Los conocimientos de los matarifes entrevistados sobre la hidatidosis/equinococosis son deficientes y las prácticas constituyen riesgo para la transmisión y diseminación de la hidatidosis.
\end{abstract}

Palabras clave Equinococosis; conocimientos, actitudes, práctica; mataderos.

Knowledge, attitudes and practices of slaughters on hydatidosis/echinococcosis in two Ica Department, Peru urban areas

\section{Abstract}

Objectives: To determine the knowledge, attitudes and practices of slaughter workers from Ica and Chincha, Ica, Peru on Echinococcus granulosus and hydatidosis/ echinococcosis. Materials and Methods: Observational, descriptive study on fifty-five workers interviewed anonymously on E. granulosus and hydatidosis/ echinococcosis in December 2000. Results: The E. granulosus metacestode was called 'water bag' by 25

1 Dirección General de Epidemiología, Ministerio de Salud. Lima, Perú.

2 Facultad de Ciencias, Universidad Nacional San Luis Gonzaga. Ica, Perú.
(45,5\%) of the workers and hydatid cysts by 10 (18,2\%). The cattle and sheep were mostly identified as intermediate hosts but the workers would not know the definitive hosts or transmission mechanisms. Thirty six $(65,5 \%)$ slaughters considered they would receive treatment if infected and 17 (30,9\%) would eliminate their dogs if they would observe helminthes in their feces stools. A group of 21 workers $(38,2 \%)$ admitted that hydatid cysts are left on the abattoir floor, $30(54,5 \%)$ raised dogs and $19(63,3 \%)$ had not given treatment for parasites to their pets in the past six months. Thirty (54,5\%) workers made clandestine cattle slaughtering and some fed their dogs with parasitized crude viscera. Conclusions: The interviewed slaughters knowledge on hydatidosis/echinococcosis was deficient and their practices favored hydatidosis transmission and spread.

Keywords: Echinococcosis; health knowledge, attitudes, practice; abattoirs. 


\section{INTRODUCCIÓN}

La hidatidosis es una zoonosis parasitaria que se adquiere por la ingestión de huevos de Echinococcus granulosus, céstode del perro y de otros cánidos silvestres. La infección humana en el Perú es considerada como uno de los problemas de salud pública en zonas ganaderas de la región andina $\left({ }^{1}\right)$. En los últimos años, se viene reportando 'casos autóctonos' en áreas urbanas no endémicas, como en la ciudad de Lima $\left({ }^{2-3}\right)$. Asimismo, se ha comunicado casos en matarifes de la ciudad de Chincha (Departamento de Ica) y se ha demostrado la infección por E. granulosus de perros vagabundos que viven cerca al camal municipal. La tasa de incidencia anual para Chincha y Pisco fue 32,0 x 100000 habitantes entre 1996 y $1998\left({ }^{4}\right)$.

Es importante determinar el nivel de conocimientos de las poblaciones en riesgo acerca de la hidatidosis/equinococosis, para implementar un programa de control. El desconocimiento permite cerrar el ciclo biológico de $E$. granulosus $\left({ }^{5}\right)$ y las creencias acerca de la forma de transmisión deben ser conocidas y tomadas en cuenta $\left(^{6}\right)$. Los hábitos y prácticas, como la matanza clandestina de animales o la alimentación de los perros con vísceras crudas parasitadas, permiten no solo mantener la enzootia, sino también, la transmisión al hombre. Los factores de riesgo asociados a los casos urbanos de hidatidosis en Lima fueron, entre otros, viajes a zonas endémicas, crianza de perros y la costumbre de dejarlos salir a la calle o alimentarlos con vísceras parasitadas $\left({ }^{3}\right)$.

En el Perú solo se ha realizado un estudio acerca de la percepción de la población general en un área endémica sobre la hidatidosis/ equinococosis $\left({ }^{7}\right)$. No existen estudios en poblaciones en alto riesgo, como los matarifes, carniceros, entre otros. Los resultados podrían orientar las estrategias de una intervención educativa. En Tunisia, solo $13,4 \%$ de 97 carniceros clandestinos entrevistados conocían el modo de transmisión $\left({ }^{8}\right)$. En Jordania, $80,6 \%$ de
144 pastores afirmaron que los perros tienen acceso a los animales beneficiados; $56,0 \%$ de los matarifes tuvieron hidatidosis; tampoco conocían bien el agente etiológico ni el hospedero definitivo $\left({ }^{9}\right)$. En la VII Región de Chile, entre las familias que sacrifican animales para consumo, casi la tercera parte de ellas alimentan a los perros con vísceras y otra proporción importante las desecha a la basura $\left({ }^{10}\right)$.

El objetivo del presente trabajo fue determinar el grado de conocimientos, actitudes y prácticas sobre $E$. granulosus y la hidatidosis/equinococosis de los matarifes de la ciudad de Ica y Chincha, específicamente el reconocimiento del quiste hidatídico y su localización, los hospederos intermediarios y definitivos, mecanismos de transmisión, síntomas y signos más frecuentes de la enfermedad, medidas de prevención y control y la posible intervención educativa de los matarifes.

\section{MATERIALES Y MÉTODOS}

La encuesta fue aplicada en Ica y en Chincha, Departamento de Ica, en la costa sur del Perú.

La ciudad de Ica se encuentra a una altitud de $406 \mathrm{msnm}$, ubicada a $300 \mathrm{~km}$ al sur de Lima. La población estimada para la provincia de Ica en el año 2000 fue 293266 habitantes $\left({ }^{11}\right)$. Las actividades económicas más importantes de la población son la agricultura y el comercio. El beneficio de animales es realizado en el camal municipal del Distrito de Ica, localizado en la zona urbana. El 3,3\% de su población tiene viviendas con características físicas inadecuadas, $9,1 \%$ viven en hacinamiento y $14 \%$ carecen de servicio de desagüe $\left({ }^{12}\right)$.

La ciudad de Chincha se encuentra a una altitud de $97 \mathrm{msnm}$, ubicada a $185 \mathrm{~km}$ al sur de Lima. La provincia de Chincha, en el año 2000, tenía una población estimada de 171709 habitantes ${ }^{(11)}$. Las actividades económicas más importantes de la población son la agricultura, la industria del agro y el comercio. El beneficio de animales 
es realizado en el camal municipal de la ciudad de Chincha, también ubicado en una zona urbana en el Distrito de Chincha Alta, que se caracteriza porque $5,9 \%$ de la población tienen viviendas precarias, $7 \%$ viviendas hacinadas y $14,1 \%$ no cuenta con servicio de desagüe $\left({ }^{12}\right)$.

La población de estudio fue los matarifes que laboraban, en los últimos seis meses al momento de la encuesta, en el camal municipal de Ica y Chincha. Estos establecimientos son los únicos mataderos oficiales en dichas provincias. Se decidió trabajar con esta población porque existía evidencia de posibles casos autóctonos de hidatidosis humana y por tener contactos para ingresar a estos establecimientos de difícil acceso.

No se calculó una muestra probabilística, debido a que no se disponía de un marco muestral; la mayoría eran trabajadores eventuales. Se entrevistó a todos los que se logró encontrar en las visitas durante el beneficio, es decir se estimó un tamaño de muestra por criterio del investigador. Para asegurarnos que la muestra de los matarifes fuera representativa, realizamos tres visitas en días diferentes al camal de la ciudad de Ica, estimándose que se entrevistó más de $90 \%$ de los trabajadores. Solamente se realizó dos visitas al camal de Chincha, porque fue difícil ingresar al establecimiento; se piensa que allí se encuestó a más de $50 \%$ de los matarifes. En estos establecimientos el beneficio se realiza solo 4 días a la semana.

Se definió como matarife a la persona que mata o descuartiza los animales en el camal o prepara los animales para consumo humano. El registro fue a través de una encuesta, corroborada por el supervisor del camal.

Se incluyó en el estudio a las personas que laboraban como matarifes en cualquier sección de beneficio animal o una actividad similar en el camal de Ica o Chincha y que hubiera laborado en los últimos seis meses al momento de la encuesta. El segundo criterio fue para asegurar la participación de la mayoría de los matarifes, debido a que este trabajo es eventual. Se excluyó a los supervisores de los matarifes, a los carniceros y a los ganaderos.

Se aplicó un cuestionario estructurado ad hoc validado, es decir que fue aplicado inicialmente en 10 matarifes para evaluar dos aspectos: la legibilidad del cuestionario y las variables que pueden ser incluidos en las preguntas. Los resultados del estudio piloto no fueron tomados en cuenta para el análisis de los resultados de la encuesta. El cuestionario aplicado tenía 21 preguntas (abiertas y cerradas) elementales sobre E. granulosus e hidatidosis/equinococosis: reconocimiento del quiste hidatídico, localización, hospederos intermediarios y definitivos, modo de transmisión, síntomas y signos de la hidatidosis humana, actitudes frente a la posible infección de sus perros con helmintos y prácticas para prevenir la infección humana y canina, posible participación en un programa de educación sanitaria. Las preguntas fueron distribuidas en tres preguntas sobre datos generales, siete sobre conocimientos básicos de la enfermedad en el hombre y en los animales, cinco acerca de las actitudes y seis sobre prácticas. Posteriormente, se retornó solamente al camal municipal de la ciudad de Ica para aplicar dos preguntas aisladas a los matarifes, relacionadas con la alimentación de sus perros.

El estudio es observacional, descriptivo, porque se describe los conocimientos, las actitudes y prácticas de los matarifes en relación a la hidatidosis/equinococosis.

La información fue recogida por alumnos del último año de biología de una universidad estatal, supervisados por un profesional. Los alumnos fueron previamente entrenados y seleccionados en la validación de la encuesta. Se aplicó entrevistas anónimas a cada trabajador, que duró entre 5 y 6 minutos, con la ayuda de muestrarios de quiste hidatídico $\left({ }^{13}\right)$. En nuestro caso, los metacestodos fueron recuperados de ovinos y colocados en frascos transparentes para mostrar a los matarifes durante la encuesta, con el fin de comprobar la certeza de las respuestas y evitar influir en los entrevistados. Las respuestas 
fueron calificadas por un investigador que conocía el tema. Para la elaboración de las tablas, se editó las respuestas para una mejor presentación.

Se elaboró una base de datos y se realizó un análisis descriptivo utilizando la frecuencia porcentual simple de las respuestas de los matarifes con la ayuda del software EPI INFO versión en español 6,04. En los casos de variables numéricas (edad de los matarifes y número de beneficios clandestinos), se calculó el promedio, la desviación estándar y la mediana.

\section{RESULTADOS}

Se entrevistó a 55 matarifes varones (37 de Ica y 18 de Chincha). La edad de los matarifes estuvo entre 18 y 66 años $(X=37,4)$, con una desviación estándar de $\pm 10,6$ años.

Respecto al grado de instrucción de los matarifes, $41(74,5 \%)$ tenían educación primaria, $13(23,7 \%)$ educación secundaria y uno $(1,8 \%)$ instrucción superior. Al analizar el área de residencia, $41(74,5 \%)$ vivían en zonas urbanas y $14 \quad(25,5 \%)$ en zonas urbanomarginales.

Las respuestas acerca de los conocimientos elementales sobre la biología de $E$. granulosus y de la hidatidosis/equinococosis fueron: 25 $(45,5 \%)$ entrevistados conocían al metacestodo de E. granulosus con el nombre de «bolsa de agua» y $10(18,2 \%)$ como quiste hidatídico; el resto no conocía o no respondió la pregunta. Treinta y siete $(67,3 \%)$ de los entrevistados conocían la localización del quiste hidatídico en los diferentes órganos de los animales. De otro lado, $19(34,5 \%)$ matarifes identificaron a los vacunos y $10(18,2 \%)$ a los ovinos como hospederos de E. granulosus (Tabla 1).

Por otro lado, $30(57,7 \%)$ de los entrevistados admitieron que el quiste hidatídico es patógeno para el hombre, pero no reconocieron los signos y síntomas más frecuentes de la enfermedad en el hombre. Detalles de los conocimientos se presenta en la Tabla 1.

Con relación a la actitud de los matarifes en el caso que estuvieran infectados con el quiste hidatídico, $36(65,5 \%)$ manifestaron su deseo de ser tratados, sin precisar el tipo de tratamiento, y $13(23,6 \%)$ acudirían al hospital para que se les practicara una cirugía. En el caso de observar helmintos en las heces de sus perros, $17(30,9 \%)$ de los matarifes los sacrificarían, $15(27,3 \%)$ los desparasitarían y $14(25,5 \%)$ los curarían o tratarían, pero no precisaron el tipo de tratamiento. Treinta y cinco $(63,6 \%)$ de los trabajadores municipales aceptarían dar una muestra de sangre para

Tabla 1. Conocimientos de los matarifes sobre Echinococcus granulosus e hidatidosis/equinococosis. Departamento de Ica, Perú.

\begin{tabular}{lrr}
\hline Respuesta & $\mathrm{n}$ & $\%$ \\
\hline Nombre del metacestodo de & & \\
E. granulosus: & & \\
- Bolsa de agua & 25 & 45,5 \\
- Quiste hidatídico & 10 & 18,2 \\
- No sabe/No respondió & 20 & 36,3 \\
El metacestodo en los animales se & & \\
localiza en: & 16 & 29,1 \\
- Vísceras & 16 & 29,1 \\
- Hígado & 5 & 9,1 \\
- Vísceras e hígado & 18 & 32,7 \\
- No sabe/No respondió & & \\
Los hospederos intermediarios del & & \\
metacestodo son: & 19 & 34,5 \\
- Vacunos & 10 & 18,2 \\
- Ovinos & 8 & 14,5 \\
- Caprinos & 4 & 7,3 \\
- Porcinos & 14 & 25,4 \\
- No sabe/No respondió & & \\
El hospedero definitivo del & 0 & 0 \\
metacestodo es: & & \\
El metacestodo es patógeno & 30 & 57,7 \\
para el hombre: & & \\
E. granulosus es trasmitido & & \\
al hombre por: & 0 & 0 \\
Los síntomas y signos más importantes & & \\
de la hidatidosis son: & & \\
- Fiebre & 10 & 18,2 \\
- Dolor del cuerpo & & \\
- No sabe/No respondió & & \\
\hline & & \\
\hline
\end{tabular}


descartar una posible infección. En el caso de iniciar un programa educativo, $53(96,4 \%)$ de los matarifes participarían en ella, $37(69,8 \%)$ preferían recibir las charlas en el camal y 14 $(26,4 \%)$ en su casa (Tabla 2$)$.

Respecto a las prácticas sobre la disposición de las vísceras contaminadas con quistes hidatídicos, $21(38,2 \%)$ admitieron que van al piso del camal y $16(29,1 \%)$, que son desechadas a la basura. Treinta $(54,5 \%)$ de los trabajadores criaban perros, de ellos 19 (63,3\%) no los habían desparasitado en los últimos seis meses. Por otro lado, treinta matarifes $(54,5 \%)$ habían beneficiado clandestinamente animales en los últimos seis meses; el número total de beneficios clandestinos fue 284 , con un rango que varió entre 1 y 50 .

Tabla 2. Actitudes de los matarifes respecto al Echinococcus granulosus y la hidatidosis/equinococosis

\begin{tabular}{lrr}
\hline Respuesta de los matarifes & $\mathrm{n}$ & $\%$ \\
\hline Actitud si le detectaran el & & \\
quiste hidatídico: & 36 & 65,5 \\
- Sería tratado & & \\
- Acudiría al hospital para & 13 & 23,6 \\
$\quad$ practicarse una cirugía: & 2 & 3,6 \\
- Acudiría a una consulta médica & 4 & 7,3 \\
- No sabe/No respondió & & \\
Actitud si su perro elimina & & \\
helmintos en sus deposiciones: & 17 & 30,9 \\
- Sacrificaría al perro & 15 & 27,3 \\
- Sería desparasitado & 14 & 25,5 \\
- Sería curado, tratado o “purgado” & 6 & 10,9 \\
- Acudiría a consulta veterinaria & 3 & 5,4 \\
- Sería abandonado & & \\
Aceptaría hacerse un análisis de & & \\
la sangre para descartar una & & \\
posible infección: & 35 & 63,6 \\
- Si & 20 & 36,4 \\
- No & & \\
Aceptaría participar de charlas & 53 & 96,4 \\
educativas sobre hidatidosis & & \\
No aceptaría participar en las & & \\
charlas educativas & & \\
Las charlas educativas las & & \\
recibirían en: & & \\
- El centro de beneficio oficial & 37 & 69,8 \\
- Su casa & & \\
- En un centro educativo & & \\
- & & \\
\hline
\end{tabular}

Los datos sobre la alimentación de los perros de los matarifes se muestra en la Tabla 3.

\section{DISCUSIÓN}

Los conocimientos, actitudes y prácticas acerca de E. granulosus y de la hidatidosis/ equinococosis en matarifes entrevistados de dos

Tabla 3. Prácticas de los matarifes respecto al Echinococcus granulosus y la hidatidosis/equinococosis.

\begin{tabular}{lll}
\hline Respuesta & $n$ & $\%$ \\
\hline
\end{tabular}

Frente al hallazgo de un quiste

hidatídico en un animal que beneficia, realiza lo siguiente:

- Es desechado al piso del matadero

- Es desechado a la basura

- Informa al veterinario sobre el hallazgo

- El quiste hidatídico es quemado

- No sabe/No respondió

Cría perros en su casa

Número de desparasitaciones de sus

perros en los últimos seis meses $(n=30)$ :

- No fue desparasitado $19 \quad 63,3$

- Una sola vez

- Dos veces

- Tres veces

De los matarifes que admitieron la

$\begin{array}{rr}21 & 38,2 \\ 16 & 29,1 \\ & \\ 6 & 10,9 \\ 4 & 7,3 \\ 8 & 14,5 \\ 30 & 54,5\end{array}$

tenencia de perros $(n=11)$,

los alimentan con*:

- Vísceras de los animales sacrificados en el camal

- Quistes hidatídicos 'bolsas de agua'

Las vísceras o los quistes hidatídicos

se los daban crudos a los perros*

16,7

13,3

6,7

Ha sacrificado animales fuera de

camal en los últimos 6 meses:

- Sí

- No

Número de beneficios clandestinos realizados por los matarifes en los últimos seis meses:

Total $=284$ Rango (1-50) Promedio $=9,46$

Mediana $=5 \mathrm{DE}=12,82$

Usa guantes para sacrificar animales en su trabajo

* Preguntas aisladas obtenidas posteriormente a la encuesta en una sola visita solo a los matarifes de Ica. $D E=$ Desviación estándar. 
zonas urbanas del Departamento de Ica, Perú, áreas consideradas hasta hace poco como no endémicas de hidatidosis, favorecen la transmisión de esta zoonosis, tal como ha sido reportado en otros países $\left({ }^{9,13,14}\right)$ y en el Perú $\left({ }^{5,7}\right)$. En la formulación de un programa de control de una enfermedad, muy poco se toma en cuenta a la persona $\left({ }^{6}\right)$. Se ha planteado que el aspecto sociocultural de la población es uno de los factores de mayor importancia en la transmisión de $E$. granulosus $\left({ }^{5}\right)$. Sus hábitos, prácticas, creencias, constituyen barreras para las actividades de prevención y control. Esta resistencia solo puede ser cambiada con una educación sanitaria que tome en cuenta al ser humano $\left(^{6}\right)$.

Los matarifes entrevistados fueron varones adultos y la mayoría con nivel de instrucción primaria. Esto podría explicar el bajo nivel de conocimientos sobre la hidatidosis/ equinococosis. Por otro lado, el trabajo que realizan los expone a mayores riesgos, comparados con una población general, para adquirir enfermedades como la hidatidosis. El papel individual de la edad y el género ha sido asociado con mayores probabilidades de contraer una enfermedad $\left({ }^{6}\right)$.

Cerca de la mitad de los matarifes conoce el metacestode como 'bolsa de agua' y un grupo muy reducido como quiste hidatídico. Las tasas de respuestas correctas son bajas comparadas con los de la población general en el Altiplano peruano $\left({ }^{5}\right)$ y áreas rurales endémicas de las provincias de Cauquenes y Linares (VII Región de Chile), que además lo conocen como 'postemas' $\left({ }^{14}\right)$, nombre que no lo hemos verificado en nuestro trabajo. Ello es explicable por el área de estudio; nuestra población vive en áreas urbanas y urbanomarginales. Se debe tomar en cuenta en las estrategias de intervención y en el diagnóstico los posibles nombres locales en las zonas ganaderas del país.

Según los matarifes, las vísceras y el hígado son los órganos más afectados por los quistes hidatídicos, resultados interesantes para el reconocimiento de los animales infectados y el manejo de las vísceras. En el sur de Jordania, la mayoría de los pastores y matarifes habían observado quistes hidatídicos en las vísceras de sus animales sacrificados $\left({ }^{9}\right)$.

Los vacunos y ovinos fueron identificados como los hospedadores intermediarios más frecuentes. Pero, llama la atención que no hayan identificado al hombre, a pesar de que lo consideran patógeno. Tampoco conocían el mecanismo de transmisión en los animales y en el hombre, que corrobora los hallazgos en Chincha, Perú $\left({ }^{4}\right)$. Éste es un ejemplo muy claro de cómo el desconocimiento del mecanismo de transmisión permite que se complete el ciclo biológico del E. granulosus $\left(^{5}\right)$. Nuestros resultados también coinciden con los reportes de áreas endémicas de Chile $\left({ }^{10}\right)$, pero difieren de otro donde $20 \%$ de personas entre infectadas y sanas conocían la forma en que los perros se infectaban $\left({ }^{14}\right)$. Se ha reportado porcentajes similares en carniceros clandestinos de Tunisia $\left({ }^{8}\right)$. En Jordania, los pastores no conocen el mecanismo de transmisión en el hombre ni en los animales, pero sí conocen los matarifes, ganaderos y las personas con hidatidosis $\left({ }^{9}\right)$. En el Altiplano peruano, $73,2 \%$ de la población general conoce el ciclo biológico $\left.{ }^{5}\right)$. Resultados que difieren de nuestros hallazgos por el área de estudio y tipo de población. Los pacientes con hidatidosis pueden conocer mejor el tema porque es posible que durante el diagnóstico hayan recibido información sobre la forma en que se habrían infectado. El conocimiento de los mecanismos de transmisión también es más elevado en áreas con programas de intervención $\left({ }^{15}\right)$.

Los matarifes entrevistados no conocían que el perro y el zorro eran los hospederos definitivos. Por ello, desconocían el papel que jugaba el perro en la transmisión de la infección al hombre en el ciclo urbano, y tampoco reconocían los síntomas y signos más importantes de la hidatidosis. Una de las estrategias claves en un programa de control para cortar la cadena de transmisión es la educación de las poblaciones endémicas $\left(^{5}\right)$ y de poblaciones en alto riesgo, como los matarifes, ganaderos, 
pastores, carniceros, quienes deben conocer el ciclo biológico de E. granulosus y la forma de transmisión de la enfermedad. La percepción en estas poblaciones debe ser explorada no solo en el ámbito del individuo, sino también, de su entorno (familia, comunidad, trabajo) $\left(^{6}\right)$.

Un número importante de trabajadores admitió que durante el beneficio el quiste hidatídico es dejado en el piso del matadero, lo que fue verificado por los investigadores, o son desechados a la basura. Son muy pocos los que lo queman, resultados que confirman las prácticas de riesgo de los matarifes de Chincha $\left({ }^{4}\right)$, los familiares de los casos índices urbanos de hidatidosis en Lima $\left({ }^{3}\right)$ y de la población general en áreas endémicas del país $\left(^{5}\right)$, Chile $\left({ }^{10,14}\right)$ y Jordania $\left({ }^{9}\right)$. Los carniceros de Tunisia también manejan inadecuadamente las vísceras parasitadas $\left({ }^{8}\right)$. El deficiente manejo de las vísceras parasitadas crudas durante el beneficio oficial por los matarifes, permitiría el acceso fácil de los perros a los quistes hidatídicos.

La mayoría de los trabajadores que encuestamos tenía entre uno o más perros. Los ganaderos los crían frecuentemente como pastores en las zonas endémicas del Perú $\left({ }^{7}\right)$. En cambio, no es frecuente que los matarifes críen perros en Jordania $\left({ }^{9}\right)$, lo que difiere de nuestros hallazgos; probablemente, los matarifes usan los caninos como guardianes. Entre los que criaban perros, la mayoría no les había administrado antihelmínticos en los últimos seis meses. Esta práctica es más frecuente que en las zonas rurales endémicas de Tunisia $\left({ }^{8}\right)$. Sin embargo, es menos frecuente que las prácticas de los pastores, ganaderos y estudiantes universitarios de Jordania $\left({ }^{9}\right)$.

La mayoría de los matarifes alimentan a sus perros con vísceras, lo que corrobora los hallazgos en Chincha, Perú $\left({ }^{4}\right)$, de zonas endémicas de Chile $\left({ }^{10}\right)$ y en poblaciones de alto riesgo en Tunisia $\left({ }^{9}\right)$. Es probable que los resultados que encontramos son muy cercanos a la verdad, debido a que se aplicó una encuesta anónima. Asimismo, coinciden con las prácticas de los familiares de los casos de hidatidosis urbana en Lima, que alimentan a sus perros con vísceras y también con los quistes hidatídicos durante los beneficios clandestinos $\left({ }^{3}\right)$. Nosotros corroboramos la costumbre de alimentar a sus perros con las "bolsas de agua". Esta práctica ha sido vista en regiones endémicas de Chile $\left({ }^{14}\right)$. En el Perú, existe la creencia de administrar las "bolsas de agua" a los perros para que sean agresivos $\left({ }^{16}\right)$. En las regiones montañosas de Marruecos, la población alimenta a sus perros con quistes hidatídicos, especialmente durante la celebración del Muslin Aid El Kebir $\left({ }^{13}\right)$, y también en zonas endémicas de Xinjiang, China $\left({ }^{17}\right)$. En una posible intervención educativa es probable que sea muy difícil evitar esta práctica. Sin embargo, esta creencia debe ser aprovechada para educar a dicha población para que alimente a sus perros con vísceras bien cocidas. En el Perú, está prohibida alimentar a los perros con desechos o productos contaminados o que ingresen a los establecimientos de salud, camales $\left({ }^{18}\right)$. Uno de los principales factores que permiten mantener la enzootia y la endemia es el acceso del perro a las vísceras parasitadas. Entonces, la principal medida de prevención y control es cortar la cadena de transmisión.

En los últimos seis meses, los matarifes habían beneficiado clandestinamente animales. Esta práctica también es frecuente en áreas endémicas del país $\left(^{5}\right)$ y en zonas urbanas de Lima $\left({ }^{3}\right)$; también es frecuente en áreas endémicas rurales de Chile $\left({ }^{10}\right)$. Los estudiantes universitarios, ganaderos y pacientes con hidatidosis en Jordania también lo practican $\left({ }^{9}\right)$. La importancia de este hallazgo es que los matarifes no solo realizan prácticas de riesgo en el centro de beneficio oficial, sino que actuarían como diseminadores de los quistes por el faenado clandestino en la población general. Se ha planteado un patrón de transmisión urbana de E. granulosus debido a las matanzas clandestinas por la población general $\left({ }^{3}\right)$. Nosotros planteamos que, en el ciclo urbano, también podrían intervenir los matarifes. La falta de tratamiento antihelmíntico de los perros, la 
práctica de matanza clandestina por los matarifes y la falta de cuidado en la alimentación de los perros podrían explicar la aparición de casos urbanos.

El hallazgo de una prevalencia de $6,5 \%$ de E. granulosus en perros vagos que se alimentan en zonas cercanas del matadero de Chincha $\left({ }^{4}\right)$, apoya la posibilidad de la transmisión urbana; en un estudio realizado en perros de una zona urbana de la ciudad de Ica, se ha reportado huevos de Taenia sp., que podría tratarse de esta especie $\left({ }^{19}\right)$.

El presente estudio exploratorio muestra limitaciones en cuanto a la validez externa e incluso interna, con número limitado de entrevistados, dificultad en acceder a esta población y no se ha incorporado otras variables, lo cual no nos permite hacer inferencias. Sin embargo, los hallazgos son importantes para enfocar los aspectos sociales de esta enfermedad y requieren ser corroborados a través de estudios epidemiológicos para identificar los factores de riesgo asociados a la infección humana. Además, conocer la magnitud de la infección humana y canina en el área urbana, incluyendo a los matarifes, carniceros; y la magnitud de la infección de los animales domésticos en las áreas urbanas y urbanomarginales donde viven los trabajadores.

Los conocimientos deficientes, las actitudes y las prácticas de los matarifes entrevistados favorecen la transmisión y diseminación de la hidatidosis humana y de la equinococosis canina. Por ello, el control y la prevención de la hidatidosis debe considerar la interrupción de la cadena para evitar que el perro pueda alimentarse de los quistes hidatídicos -estrategia clave-, el control del beneficio de los animales en los centros oficiales de beneficio $\left({ }^{20}\right)$ y el manejo adecuado de los residuos, la educación sanitaria de la población en riesgo y de alto riesgo sobre el ciclo biológico de la tenia y el mecanismo de transmisión. Asimismo, su participación para lograr el cambio de actitudes y las prácticas con un enfoque que piensa en el individuo. Y, finalmente, la supervisión por los municipios para el cumplimiento de las normas establecidas para la crianza adecuada de los perros.

\section{AGRADECIMIENTOS}

A E. Maldonado por haber participado en la encuesta y al Dr. Pedro Cabrera por las facilidades brindadas para realizar el estudio.

\section{REFERENCIAS BIBLIOGRÁFICAS}

1. Moro PL, McDonald J, Gilman RH, Silva B, Verastegui M, Malqui V, et al. Epidemiology of Echinococcus granulosus infection in the central Peruvian Andes. Bull World Health Organiz. 1997;75(5):553-61.

2. Alarcón J, Somocurcio J, Piscoya J, Reyes N, Arévalo N, Bustamante E. Hidatidosis pulmonar: estudio epidemiológico de casos urbanos en el Hospital Hipólito Unanue de Lima. Rev Peru Epidemiol. 1992;5(2):15-9.

3. Huamán IMR. Identificación de la cadena de transmisión de casos autóctonos de hidatidosis urbana. [Tesis para optar el título de Biólogo]. Lima: Facultad de Ciencias Biológicas, Universidad Nacional Mayor de San Marcos; 1999.

4. Moro PL, Lopera L, Cabrera M, Cabrera G, Silva B, Gilman RH, et al. Short report: endemic focus of cystic echinococcosis in a coastal city of Peru. Am J Trop Med Hyg. 2004;71(3):327-9.

5. Rojas CM. Parasitismo de los rumiantes domésticos. Terapia, prevención y modelos para su aprendizaje. Lima: Editorial Maijosa; 1990.

6. Briceño-León R. Siete tesis sobre la educación sanitaria para la participación comunitaria. Cad Saúde Públ. 1996;12(1):730.

7. Moro PL, Lopera L, Bonifacio N, Gonzales A, Gilman $\mathrm{RH}$, Moro MH. Risk factors for canine echinococcosis in an endemic area of Peru. Vet Parasitol. 2005;130(1-2):99104.

8. Besbes M, Sellami M, Cheickhrouhou F, Makni F, Ayadi A. Clandestine slaughtering in Tunisia: investigation on the knowledge and practices of butchers concerning hydatidosis. Bull Soc Pathol Exot. 2003;96(4):320-2.

9. Nasrieh MA, Abdel-Hafez SK, Kamhawi SA, Craig PS, Schantz PM. Cystic echinococcosis in Jordan: socioeconomic evaluation and risk factors. Parasitol Res. 2003;90:456-66.

10. Apt W, Pérez C, Galdamez E, Campano S, Vega F, Vargas $\mathrm{O}$, et al. Equinococosis/ hidatidosis en la VII Región de Chile: diagnóstico e intervención educativa. Rev Panam Salud Pública. 2000;7:8-16. 
11. Instituto Nacional de Estadística e Informática, Dirección Técnica de Demografía e Indicares Sociales. Perú: proyecciones de población, por años calendario según departamentos, provincias y distritos (Período, 1990-2005). Boletín Especial No 16. Lima: Instituto Nacional de Estadística e Informática; 2002.

12. Instituto Nacional de Estadística e Informática, Dirección Técnica de Demografía y Estudios Sociales - Fondo de Población de las Naciones Unidas. Perú: mapa de necesidades básicas insatisfechas de los hogares a nivel distrital. Lima: Instituto Nacional de Estadística e Informática, Fondo de Población de las Naciones Unidas; 1994.

13. Kachani M, Macpherson CNL, Lyagoubi M, Berrada M, Bouslikhane M, Kachani F, et al. Public health education/ importance and experience from the field. Educational impact of community-based ultrasound screening surveys. Acta Trop. 2003;85:263-9.

14. Galdamez D, Cortes P, Vargas D, Rodríguez J, Vega F, Perez C, et al. Variables epidemiológicas asociadas a hidatidosis en población rural asintomática. Parasitol al Día. 1997;21(1-2):7-13.

15. Yamamoto N, Kishi R, Miyake H. A comparative study of public knowledge and awareness of echinococcus in relation to anti-echinococcus polices of towns in Hokkaiddo. Nippon Koshu Eisei Zasshi. 1995;42:559-68.

16. Elliot A, Cáceres I. Introducción a la parasitología médica del Perú. Lima: Martegraf; 1994.
17. Craig PS, Deshan L, Zhaoxun D. Hydatid disease in China. Parasitol Today. 1991;7(1):46-50.

18. Aprueban el reglamento de la Ley que regula el régimen jurídico de canes. Decreto Supremo No 006-2002-SA. El Peruano 2002 Jun 25; Secc. Salud. p.225240-4.

19. Trillo-Altamirano MDP, Carrasco TAJ, Cabrera R. Prevalencia de helmintos enteroparásitos zoonóticos y factores asociados en Canis familiaris en una zona urbana de la ciudad de Ica, Perú. Parasitol Latinoam. 2003;58:13641.

20. Rojas CM. Cisticercosis e hidatidosis: metacestodiasis de perentorio control en el Perú. Rev virt parasit vet peru [serial en la Internet]. 2002 Jun [citado 2005-04-06];1(1): [aprox. 15 p.]. Disponible en: http://www.visionveterinaria.com/ rojas/cisticercosis01.htm

Manuscrito recibido el 06 de setiembre de 2005 y aceptado para publicación el 30 setiembre de 2005.

Correspondencia: Blgo. Rufino Cabrera

Dirección General de Epidemiología, Ministerio de Salud. Camilo Carrillo 402

Lima 11, Perú.

Correo-e: rcabrerach@hotmail.com 